\title{
Kinematic analysis of a novel 3-CRU translational parallel mechanism
}

\author{
B. Li ${ }^{1,2}$, Y. M. Li ${ }^{1,2}$, X. H. Zhao ${ }^{2}$, and W. M. Ge ${ }^{2}$ \\ ${ }^{1}$ Faculty of Science and Technology, University of Macau, Taipa, Macau, China \\ ${ }^{2}$ Tianjin Key Laboratory for Advanced Mechatronic System Design and Intelligent Control, Tianjin University \\ of Technology, Tianjin, China \\ Correspondence to: Y. M. Li (ymli@umac.mo)
}

Received: 25 November 2014 - Revised: 1 April 2015 - Accepted: 12 April 2015 - Published: 24 April 2015

\begin{abstract}
In this paper, a modified 3-DOF (degrees of freedom) translational parallel mechanism (TPM) threeCRU (C, R, and U represent the cylindrical, revolute, and universal joints, respectively) structure is proposed. The architecture of the TPM is comprised of a moving platform attached to a base through three CRU jointed serial linkages. The prismatic motions of the cylindrical joints are considered to be actively actuated. Kinematics and performance of the TPM are studied systematically. Firstly, the structural characteristics of the mechanism are described, and then some comparisons are made with the existing 3-CRU parallel mechanisms. Although these two 3-CRU parallel mechanisms are both composed of the same CRU limbs, the types of freedoms are completely different due to the different arrangements of limbs. The DOFs of this TPM are analyzed by means of screw theory. Secondly, both the inverse and forward displacements are derived in closed form, and then these two problems are calculated directly in explicit form. Thereafter, the Jacobian matrix of the mechanism is derived, the performances of the mechanism are evaluated based on the conditioning index, and the performance of a 3-CRU TPM changing with the actuator layout angle is investigated. Thirdly, the workspace of the mechanism is obtained based on the forward position analysis, and the reachable workspace volume is derived when the actuator layout angle is changed. Finally, some conclusions are given and the potential applications of the mechanism are pointed out.
\end{abstract}

\section{Introduction}

In recent years, lower-mobility translational parallel mechanisms (TPMs) have been extensively studied. Compared with 6-DOF (degrees of freedom) parallel manipulators, lowermobility mechanisms possess many merits in terms of simpler mechanical design, larger workspace, and lower manufacturing cost, in addition to the inherent advantages of the general parallel manipulators, such as high accuracy, high stiffness, high velocity, high dynamic performance, large load-to-weight ratio, low moving inertia, and little accumulation of positional errors. A lower-mobility TPM with 3 DOFs is a focus of the current trend in the research community, and various forms of 3-DOF TPMs have been designed. A 3-DOF TPM is a 3-DOF parallel mechanism whose moving platform can achieve three independent orthogonal translational motions with respect to its fixed base.
In past related research literature and industrial applications, the well-known 3-DOF TPM Delta robot (Clavel, 1988), one of the most successful parallel manipulators in market, was commonly used in pick-and-place applications. A pure translational 3-UPU PM (P represents the prismatic joint) was proposed in Tsai and Joshi (2000); this mechanism has been the subject of much study and was previously widely used in practice, and several variants of this mechanism were designed, such as 3-PUU TPM and the FlexPLP tripod. A translational 3-URC mechanism was proposed in Gregorio (2004); the position and velocity of this PM were written in explicit form. A kind of 3-DOF translational parallel cube manipulator was presented in the study of $\mathrm{Li}$ et al. (2003), in which the kinematics and workspace of the manipulator were investigated. Compared with other 3-DOF TPMs, the parallel cube manipulator possessed some obvious merits 


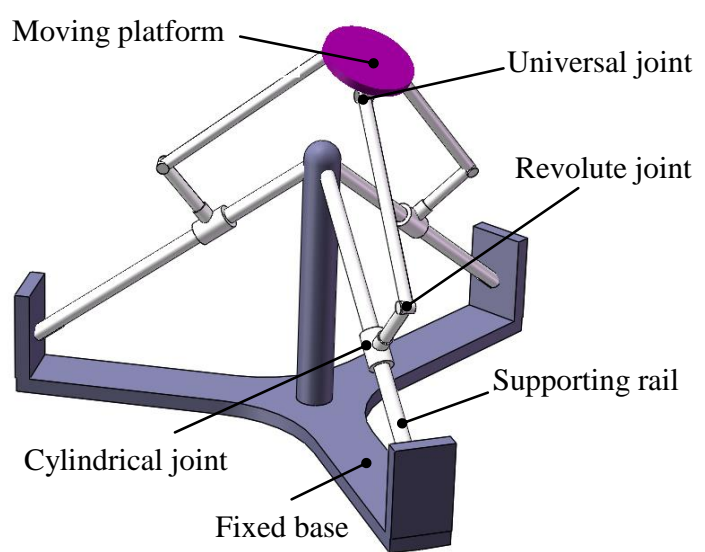

Figure 1. The existing 3-CRU spherical wrist.

in terms of higher compactness and stiffness and no singularities in the workspace. Lou and $\mathrm{Li}$ (2006) proposed a novel 3DOF purely TPM, named Orthotripod. The mechanism possessed a nearly ball-shaped workspace, and had a much better conditioning index than that of the tripod-based mechanism. The CICABOT was presented in Ruiz et al. (2012), which was a novel 3-DOF TPM based on two five-bar mechanisms. The workspace of the CICABOT was large and its workspace volume was limited by the size of the links, and both the inverse and direct kinematics were very simple to determine. The dynamics modeling of a translational 3-CPU was investigated via screw theory (Carbonari et al., 2013). The operation performance of a LARM leg mechanism with 3-UPU parallel architecture was studied in Wang et al. (2015). Besides these new 3-DOF TPMs mentioned above, some other 3-DOF architectures can be found in the literature ( $\mathrm{Li}$ and Xu, 2006; Simoni et al., 2013; Gregorio and Parenti-Castelli, 2002; Ji and Wu, 2003; Chung and Hervé, 2006; Kim and Chung, 2003; Merlet, 2006; Li and Xu, 2005a, b; Tsai and Joshi, 2002; Chablat and Wenger, 2001).

In this paper, a modified 3-DOF TPM (3-CRU, where the letters $\mathrm{C}, \mathrm{R}$, and $\mathrm{U}$ represent the cylindrical, revolute, and universal joints, respectively) is proposed. The structure of the paper is arranged as follows. In Sect. 2, the proposed mechanism is compared with other existing mechanisms. The mobility of the mechanism is analyzed by means of screw theory in Sect. 3. In Sect. 4, an analytical model for the kinematics of the mechanism is established, and the exact analytical solutions are found both for the inverse and forward kinematics problems. In Sect. 5, the Jacobian matrix of the mechanism is derived. The reachable workspace of the mechanism is obtained based on the forward position analysis in Sect. 6. Conclusions and areas of future research are given in Sect. 7.

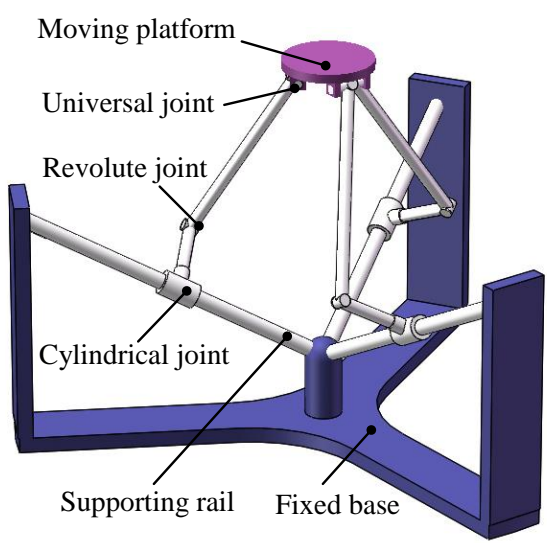

Figure 2. The modified 3-CRU TPM.

\section{A modified 3-CRU TPM and its structural characteristics}

With regard to previous works, a 3-CRU rotational parallel manipulator, as shown in Fig. 1, was first introduced in Fang and Tsai (2004); subsequent to this, the kinematics, dynamics and kineto-elasto-static synthesis of a 3-CRU spherical wrist were studied by Callegari et al. (2007a, b).

In this paper, a modified structure of 3-CRU TPM, shown in Fig. 2, is proposed. The orientational mechanism (Fig. 1) and the positional mechanism (Fig. 2) differ from each other in the axes of the revolute joints and universal joints. The different joint arrangements of CRU limbs in these two types of 3-CRU parallel mechanisms are demonstrated in Fig. 3. The 3-CRU TPM consists of a base platform, a moving platform, three supporting rails, and three limbs with identical kinematic structures. Each limb connects the fixed base to the moving platform via a cylindrical joint, a revolute joint, and a universal joint in sequence. The cylindrical joint, actuated by a linear actuator, can move along the supporting rail and rotate on the rail simultaneously (Fig. 4), and the rails are symmetrically arranged $120^{\circ}$ apart. Thus, the moving platform is attached to the base by three identical CRU linkages.

For the sake of analysis, a fixed Cartesian reference coordinate frame $O-x y z$ is attached at the centered point $O$ of the intersection point of three supporting rails as shown in Fig. 5. $A_{i}$ is the center of the cylindrical joint, $B_{i}$ is the center of the revolute joint, $C_{i}$ is the center of the universal joint, and point $P$ is the center of the moving platform. Angle $\alpha_{i}$ is measured from the base platform to rails $O A_{i}$ and is defined as the actuator layout angle. In order to ensure the isotropic property of the mechanism, we assume $\alpha_{1}=\alpha_{2}=\alpha_{3}=\alpha$.

\section{Mobility analysis via screw theory}

Considering that the general Grübler-Kutzbach criterion can only obtain the number of DOFs for some mechanisms but cannot indicate the properties of the DOF (i.e., whether they 


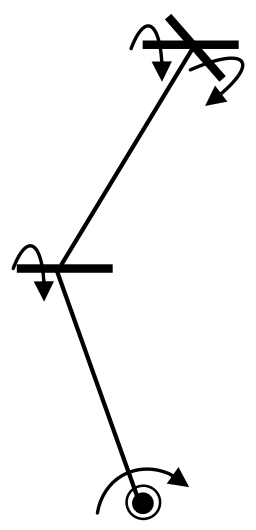

(a)

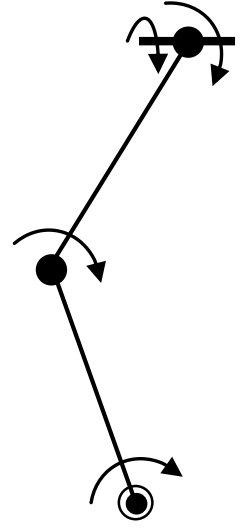

(b)
Revolute joint

Cylindrical joint

Figure 3. Different joint arrangement of a CRU limb. (a) Joint arrangement of the CRU limb of an existing 3-CRU spherical wrist. (b) Joint arrangement of a CRU limb of a modified 3-CRU TPM.

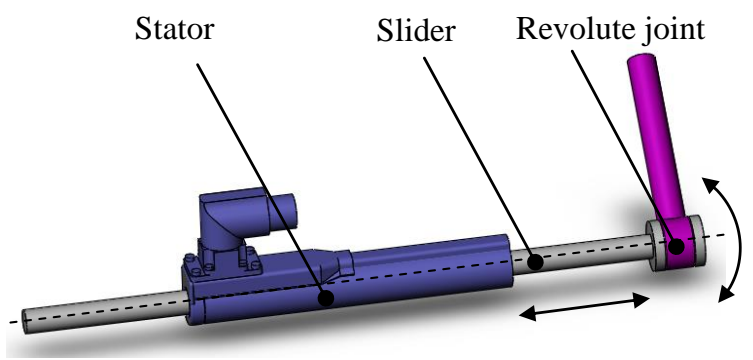

Figure 4. A CAD model of a cylindrical joint.

are translational or rotational DOF), screw theory is employed to analyze the mobility of a 3-CRU parallel manipulator, which is a convenient tool for studying instantaneous motion systems that include both rotational and translational motions in three-dimensional space.

The mobility of the 3-CRU TPM is determined by the combined effect of the three limb constraint forces/couples. Here, the reciprocal screw theory is used to analyze constraint forces exerted on the moving platform in order to give a complete description of how the mobility of TPMs is computed (Dai et al., 2006). Without losing generality, a local coordinate system $A_{i}-x_{i} y_{i} z_{i},(i=1-3)$ is established for each limb and twist system of the $i$ th CRU limb as shown in

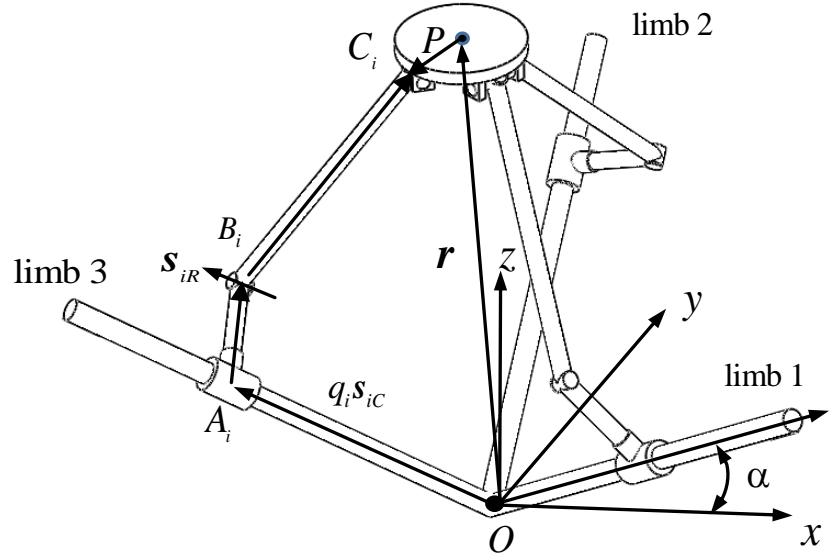

Figure 5. Schematic model of the mechanism.

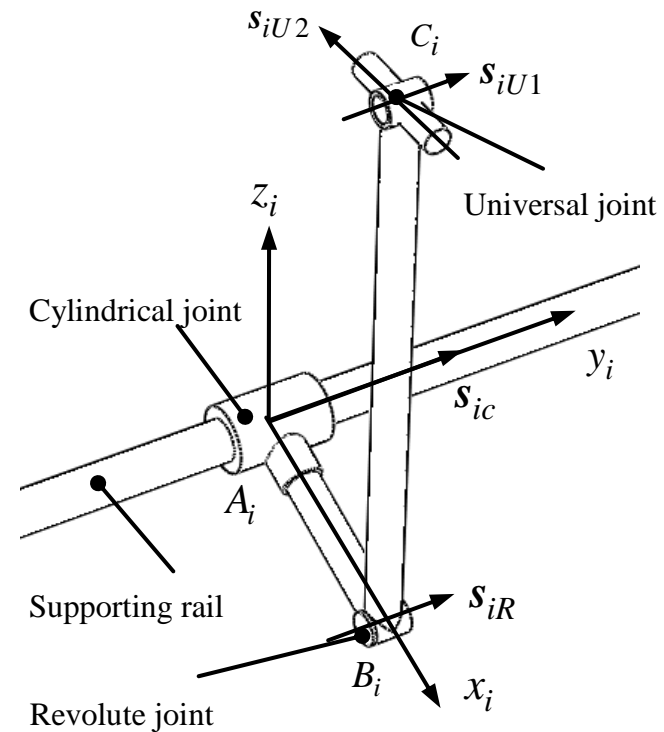

Figure 6. Twist systems of a CRU limb.

Fig. 6:

$$
\begin{aligned}
& \$_{1}=\left(\begin{array}{ll}
\mathbf{0} & \boldsymbol{s}_{i \mathrm{C}}
\end{array}\right) \\
& \$_{2}=\left(\begin{array}{ll}
s_{i \mathrm{C}} & \mathbf{0}
\end{array}\right) \\
& \$_{3}=\left(\begin{array}{ll}
s_{i \mathrm{R}} & \boldsymbol{r}_{i \mathrm{R}} \times s_{i \mathrm{R}}
\end{array}\right) \\
& \$_{4}=\left(\begin{array}{ll}
s_{i \mathrm{U} 1} & \boldsymbol{r}_{i \mathrm{U}} \times s_{i \mathrm{U} 1}
\end{array}\right) \\
& \$_{5}=\left(\begin{array}{ll}
\boldsymbol{s}_{i \mathrm{U} 2} & \boldsymbol{r}_{i \mathrm{U}} \times s_{i \mathrm{U} 2}
\end{array}\right),
\end{aligned}
$$

where $s_{i \mathrm{C}}$ and $s_{i \mathrm{R}}$ stand for the unit direction vector of cylindrical and revolute joints, respectively, and $s_{i \mathrm{C}}=s_{i \mathrm{R}} \cdot \boldsymbol{s}_{i \mathrm{U} 1}$ and $s_{i \mathrm{U} 2}$ are the unit direction vectors of universal joints.

Using the reciprocity between twist and wrench, the CRU limb constraint system can be calculated by

$\$_{1}^{r}=\left(\begin{array}{ll}\mathbf{0} & \boldsymbol{n}_{i}\end{array}\right)$,

where $\boldsymbol{n}_{i}=\boldsymbol{s}_{i \mathrm{U} 1} \times \boldsymbol{s}_{i \mathrm{U} 2} . \$_{1}^{r}$ denotes a constraint couple whose direction is perpendicular to the axes of joint screws $\$_{4}$ and $\$ 5$. 


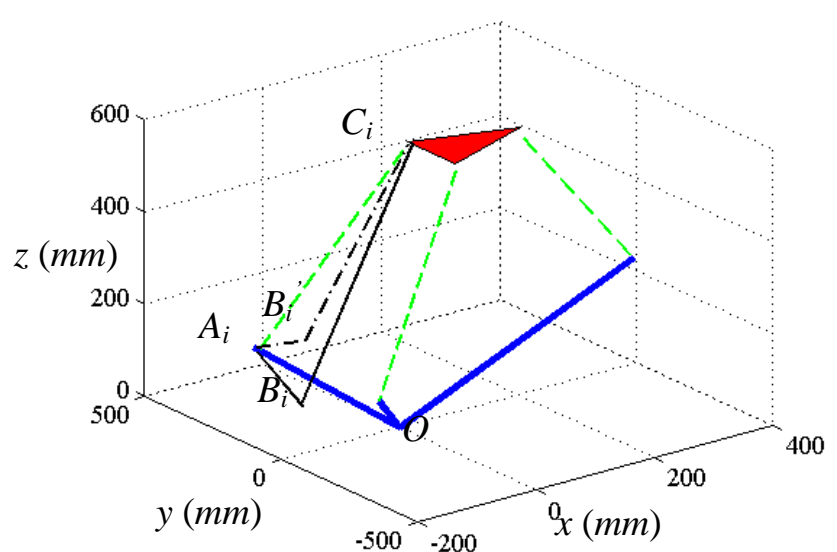

Figure 7. Different configurations for inverse position.

In Eq. (2), three CRU limbs provide three constraint couples restricting rotational DOFs around the axis of the constraint couples. Therefore the moving platform of the 3-CRU TPM behaves as if it has only three translational DOFs.

\section{Inverse and forward position analyses}

\subsection{Inverse position analysis}

Inverse position analysis of the 3-CRU TPM involves the determination of the position of $A_{i}$ given the position of the moving platform.

In the $O-x y z$ frame, the position vector $\boldsymbol{r}=(x y z)^{T}$ of position $P$ can be expressed as

$\boldsymbol{r}=q_{i} \boldsymbol{s}_{i \mathrm{C}}+\overline{\boldsymbol{A}_{i} \boldsymbol{B}_{i}}+\overline{\boldsymbol{B}_{i} \boldsymbol{C}_{i}}-\overline{\boldsymbol{P} \boldsymbol{C}_{i}}, \quad(i=1,2,3)$,

where $q_{i}=\left\|O A_{i}\right\|$ stands for the position of $A_{i}, s_{i \mathrm{C}}$ is the unit vector of $\overline{O A_{i}}, \overline{\boldsymbol{A}_{i} \boldsymbol{B}_{i}}$ is the vector from point $A_{i}$ to point $B_{i}, \overline{\boldsymbol{B}_{i} \boldsymbol{C}_{i}}$ is the vector from point $B_{i}$ to point $C_{i}$, and $\overline{\boldsymbol{P \boldsymbol { C } _ { i }}}$ is the vector from point $P$ to point $C_{i}$.

Note that, for the CRU limb, the constraint imposed by the revolute joint restricts both $\overline{\boldsymbol{A}_{i} \boldsymbol{B}_{i}}$ and $\overline{\boldsymbol{B}_{i} \boldsymbol{C}_{i}}$ so that they are normal to the unit vector $s_{i \mathrm{R}}$ of the revolute joint axis. Thus, taking the dot product with $s_{i \mathrm{R}}$ on both sides of Eq. (3) leads to

$q_{i}=\left(\boldsymbol{r}+{\overline{P C_{i}}}^{T} \boldsymbol{s}_{i \mathrm{R}}, \quad(i=1,2,3)\right.$.

Thus, for a given position vector $\boldsymbol{r}=\left(\begin{array}{lll}x & y & z\end{array}\right)^{T}$ of the moving platform, the position of $A_{i}$ can be obtained directly using Eq. (4).

\subsection{Forward position analysis}

Forward position analysis of the mechanism is concerned with the determination of the moving platform position given the position of $A_{i}$.
Expanding Eq. (4) yields

$q_{1}=\cos (\alpha) x+\sin (\alpha) z+\cos (\alpha) a$,

$q_{2}=-\frac{1}{2} \cos (\alpha) x+\frac{\sqrt{3}}{2} \cos (\alpha) y+\sin (\alpha) z+\cos (\alpha) a$,

$q_{3}=-\frac{1}{2} \cos (\alpha) x-\frac{\sqrt{3}}{2} \cos (\alpha) y+\sin (\alpha) z+\cos (\alpha) a$.

Subtracting Eq. (7) from Eq. (6) yields

$y=\frac{q_{2}-q_{3}}{\sqrt{3} \cos (\alpha)}$.

Adding Eqs. (5), (6), and (7) together yields

$z=\frac{q_{1}+q_{2}+q_{3}-3 \cos (\alpha) a}{3 \sin (\alpha)}$.

Then, substituting Eq. (9) into Eq. (5) gives

$x=\frac{2 q_{1}-q_{2}-q_{3}}{3 \cos (\alpha)}$.

Lastly, given a set of ( $\left.q_{1} q_{2} q_{3}\right), x, y$, and $z$ can be solved by using Eqs. (8), (9), and (10).

It should be pointed out that the actuator layout angle should be set in the range of $0-90^{\circ}$ to ensure that the robot has real solutions for the forward position analysis.

For the proposed 3-CRU TPM in this paper, both the inverse and the forward position analyses of the mechanism can be calculated directly in explicit form as shown in Sects. 4.1 and 4.2, which is extremely significant for the possible practical applications of the mechanism.

\subsection{Numerical examples}

The architectural parameters of a 3-CRU TPM are selected as $a=100 \mathrm{~mm}, l_{1}=300 \mathrm{~mm}, l_{2}=500 \mathrm{~mm}$, and $\alpha=30^{\circ}$; here, $a=\left\|\boldsymbol{P} \boldsymbol{C}_{i}\right\|, l_{1}=\left\|\boldsymbol{A}_{i} \boldsymbol{B}_{i}\right\|$, and $l_{2}=\left\|\boldsymbol{B}_{i} \boldsymbol{C}_{i}\right\|$. For the inverse position analysis, given a set of inputs $\left(\begin{array}{lll}x & y & z\end{array}\right)$, output parameters can be calculated as shown in Table 1, and Fig. 7 depicts the configurations associated with these solutions. For the forward position analysis, given a set of inputs $\left(\begin{array}{lll}q_{1} & q_{2} & q_{3}\end{array}\right)$, output parameters can be calculated as shown in Table 2 , and the configurations associated with these solutions are described in Fig. 8.

For a given position vector $r=\left(\begin{array}{lll}x & y & z\end{array}\right)^{T}$ of the moving platform, only one solution of the $A_{i}$ positions can be obtained using Eq. (4) directly, as shown in Fig. 7. However, the locations of point $B_{i}$ have two possibilities for a CRU limb, as shown in Fig. 9. Therefore, there are in total eight configurations for the inverse position analysis. Accordingly, the forward position analysis also has eight solutions in a similar manner. But for the 3-CRU orientation parallel mechanism, each limb has four feasible solutions, leading to a total of 64 possibilities for the inverse and forward position analyses (Callegari et al., 2007b). Obviously, the kinematics of the modified positional parallel mechanism 3-CRU are much simpler than those of the orientational mechanism. 
Table 1. Inverse position analysis of the mechanism (unit: $\mathrm{mm}$ ).

\begin{tabular}{rrrrrrrrr}
\hline & \multicolumn{1}{c}{ Inputs } & & & \multicolumn{2}{c}{ Outputs } & & \\
\cline { 2 - 4 } \cline { 6 - 8 } & $x$ & $y$ & $z$ & & $q_{1}$ & $q_{2}$ & $q_{3}$ \\
\hline Case (a) & 80 & -50 & 600 & & 455.8846 & 314.4615 & 389.4615 \\
\hline
\end{tabular}

Table 2. Forward position analysis of the mechanism (unit: $\mathrm{mm}$ ).

\begin{tabular}{rrrrrrrrr}
\hline & \multicolumn{3}{c}{ Inputs } & & & & Outputs & \\
\cline { 2 - 4 } \cline { 6 - 8 } & $q_{1}$ & $q_{2}$ & $q_{3}$ & & $x$ & $y$ & $z$ \\
\hline Case (b) & 300 & 500 & 500 & & -153.9601 & 0 & 693.4616 \\
\hline
\end{tabular}

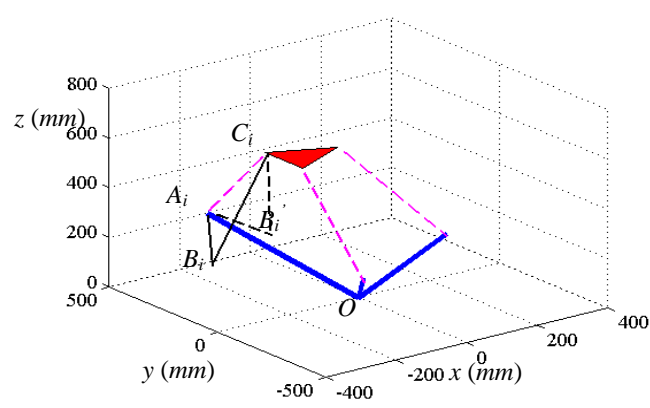

Figure 8. Different configurations for forward position analysis.

\section{Jacobian matrix of the mechanism and performance analysis}

\subsection{Jacobian matrix of the mechanism}

Differentiating Eqs. (5), (6), and (7) with respect to time respectively yields

$\dot{q}_{1}=\cos (\alpha) \dot{x}+\sin (\alpha) \dot{z}$,

$\dot{q}_{2}=-\frac{1}{2} \cos (\alpha) \dot{x}+\frac{\sqrt{3}}{2} \cos (\alpha) \dot{y}+\sin (\alpha) \dot{z}$,

$\dot{q}_{3}=-\frac{1}{2} \cos (\alpha) \dot{x}-\frac{\sqrt{3}}{2} \cos (\alpha) \dot{y}+\sin (\alpha) \dot{z}$.

Equations (11), (12), and (13) can be written in matrix form. Only when the manipulator is away from singularities is the matrix invertible.

$\dot{\boldsymbol{q}}=\mathbf{J} \dot{\boldsymbol{X}}$,

where $\dot{\boldsymbol{q}}=\left[\begin{array}{lll}\dot{q}_{1} & \dot{q}_{2} & \dot{q}_{3}\end{array}\right]^{T}, \dot{q}_{i}$ is the velocity of the $i$ th linear actuator, and $\dot{X}=\left[\begin{array}{ccc}\dot{x} & \dot{y} & \dot{z}\end{array}\right]^{T}$ represents the threedimensional linear velocity of the moving platform.

$\mathbf{J}=\left[\begin{array}{ccc}\cos (\alpha) & 0 & \sin (\alpha) \\ -\frac{1}{2} \cos (\alpha) & \frac{\sqrt{3}}{2} \cos (\alpha) & \sin (\alpha) \\ -\frac{1}{2} \cos (\alpha) & -\frac{\sqrt{3}}{2} \cos (\alpha) & \sin (\alpha)\end{array}\right]$

is the Jacobian matrix of the mechanism.

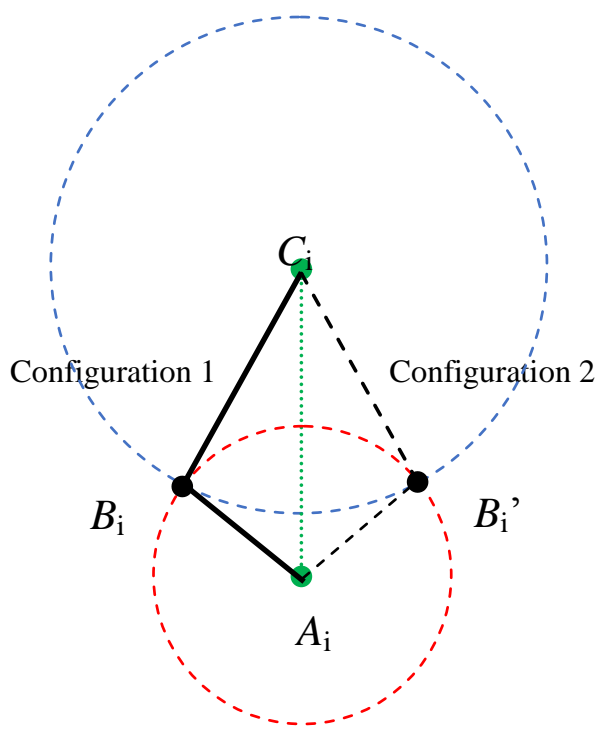

Figure 9. Two possible configurations of a CRU limb.

When $\mathbf{J}$ is invertible, Eq. (14) can be written as

$\dot{\boldsymbol{X}}=\mathbf{J}^{-1} \dot{\boldsymbol{q}}$

Equation (15) represents the forward velocity solution for a 3-CRU TPM.

\subsection{Performance analysis}

With respect to performance evaluation and optimization, the most used parameter is the Jacobian matrix, which is the matrix map of the velocity of the end effector onto the vector of actuated joint velocities. The conditional number of the Jacobian matrix, called the local conditioning index (LCI) (Li et al., 2005), was applied for performance evaluation of parallel manipulators. The conditioning index can be defined as the ratio of the smallest $\lambda_{\min }$ to the largest $\lambda_{\max }$ singular values of $\mathbf{J}$, i.e.,

$\kappa=\frac{\lambda_{\min }}{\lambda_{\max }}$.

For the proposed 3-CRU TPM, according to Eq. (14), the conditioning index of the mechanism is only related to the actuator layout angle $\alpha$. Naturally, how the output characteristics of a 3-CRU TPM vary with differences in actuator layout angle is studied. The mechanical parameters of the 


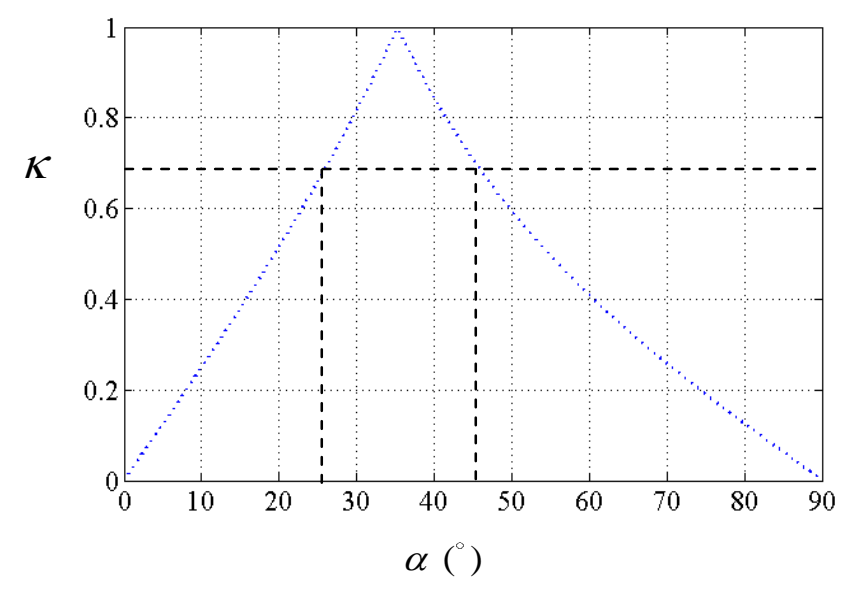

Figure 10. Relationship between the performance and the actuator layout angle.

mechanism are set up in the same way as those in Sect. 4.3. Then, the relationship between the performance of the mechanism and the actuator layout angle $\alpha$ is obtained as shown in Fig. 10, and the actuator layout angle should be given in the range $25-45^{\circ}$ to ensure good kinematic performance of the manipulator.

\section{Workspace analysis}

In this section, the reachable workspace of the 3-CRU TPM is obtained based on the forward position analysis. Given a set of limb lengths ( $\left.q_{1} q_{2} q_{3}\right)$, the position of the moving platform can be calculated directly by corresponding equations as shown in Section 4. Thus when the restrictions to the limb lengths are set up, the reachable workspace of the mechanism can be obtained. It should be mentioned that few TPMs can obtain the workspace through forward position analysis; this is also one novel contribution in this paper.

Compared with serial ones, parallel manipulators have a relatively small workspace. The characteristics change with the variation in the actuator layout angle in the reachable workspace of a 3-CRU TPM..

The restrictions to the limb lengths are defined as $300 \mathrm{~mm} \leq q_{1} \leq 600 \mathrm{~mm}, \quad 300 \mathrm{~mm} \leq q_{2} \leq 600 \mathrm{~mm}$, and $300 \mathrm{~mm} \leq q_{3} \leq 600 \mathrm{~mm}$. The workspace of the manipulator can be generated by a MATLAB program, the results of which are shown in Fig. 11.

In order to investigate the reachable workspace volume of a 3-CRU TPM with the changing of the actuator layout angle, the workspace volume is illustrated in Fig. 12, from which it can be observed that the maximum workspace volume occurs when the actuator layout angle $\alpha$ is around $35^{\circ}$. The $x-y$ section of the workspace for $\alpha=35^{\circ}$ is shown in Fig. 13.
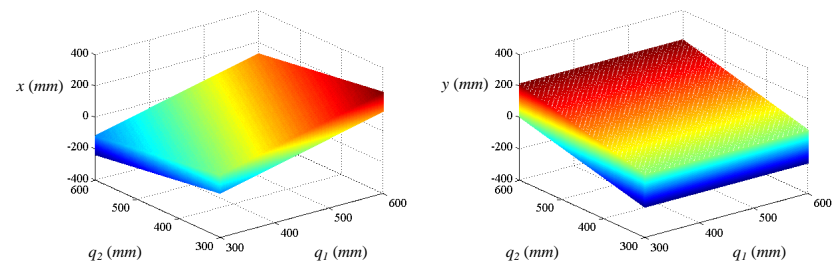

(a)
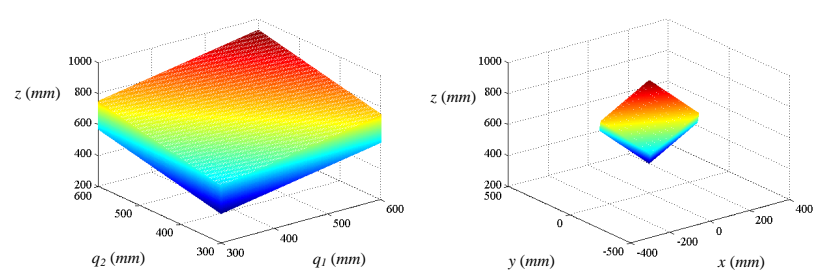

(c)

(d)

Figure 11. The workspace of the manipulator.

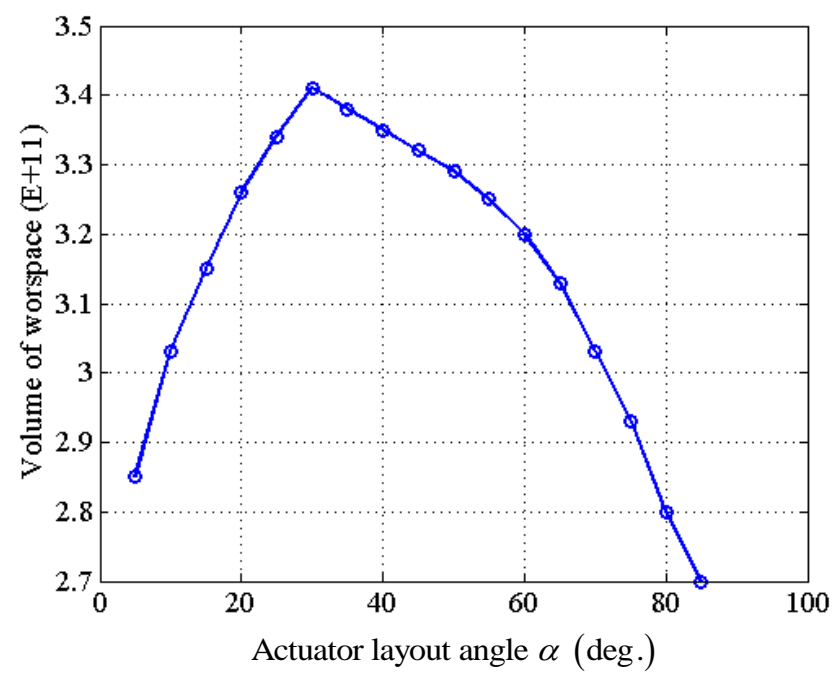

Figure 12. Reachable workspace volume versus actuator layout angle.

\section{Conclusions}

This paper proposes a modified 3-DOF TPM 3-CRU. The mobility of the mechanism is analyzed based on screw theory. Each CRU limb exerts one constraint couple on the platform. Both inverse and forward position analyses are performed, and the analytical solutions are obtained with respect to these two problems. Unlike most parallel robots, the proposed TPM has explicit solutions for inverse and forward kinematics issues. Therefore, both the path planning and control problems of the mechanism are very simple. Additionally, the Jacobian matrix of the mechanism is obtained, the performance is evaluated through a conditioning index, and the performance of a 3-CRU TPM along with the various actuator layout angles is investigated. Furthermore, the 


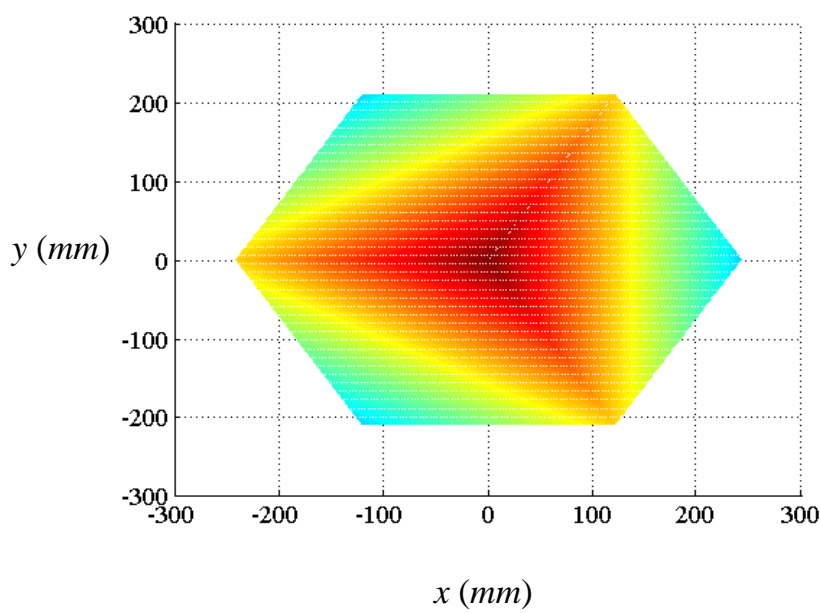

Figure 13. $x-y$ section of the workspace at $\alpha=35^{\circ}$.

reachable workspace is obtained based on the forward position analysis, and the reachable workspace volume is obtained when the actuator layout angle is varied. On the basis of the kinematics analysis of the mechanism, analyses of inverse/forward dynamics and stiffness performance as well as kinematic optimization of the mechanism will be investigated in future work.

Acknowledgements. This work was supported by the National Natural Science Foundation of China (51205289, 51275353), the Natural Science Foundation of Tianjin (14JCZDJC39100), the Macao Science and Technology Development Fund (108/2012/A3, 110/2013/A3), and the Research Committee of University of Macau (MYRG183(Y1-L3)FST11-LYM, MYRG203(Y1-L4)FST11-LYM).

Edited by: J. Schmiedeler

Reviewed by: two anonymous referees

\section{References}

Callegari, M., Cammarata, A., Gabrielli, A., and Sinatra, R.: Kinematics and dynamics of a 3-CRU spherical parallel robot, in: Proceedings of the ASME International Design Engineering Technical Conferences \& Computers and Information in Engineering Conference, Las Vegas, Nevada, USA, 91-101, 2007.

Callegari, M., Cammarata, A., and Ruggiu, M.: Kinetostatic synthesis of a 3-CRU spherical wrist for miniaturized assembly tasks, Proc. 18th AIMeTA Congress of Theoretical and Applied Mechanics, Brescia, Italy, 10-26, 2007.

Carbonari, L., Battistelli, M., Callegari, M., and Palpacelli, M.-C.: Dynamic modelling of a 3-CPU parallel robot via screw theory, Mech. Sci., 4, 185-197, doi:10.5194/ms-4-185-2013, 2013.

Chablat, D. and Wenger, P.: Architecture optimization of a 3-DOF translational parallel mechanism for machining applications, the Orthoglide, IEEE Trans. Robot. Auto., 255, 1-8, 2001.
Chung, C. L. and Hervé, J. M.: Translational parallel manipulators with doubly planar limbs, Mech. Mach. Theory, 41, 433-455, 2006.

Clavel, R.: Delta, A fast robot with parallel geometry, in: Proceedings of 18th International Symposium on Industrial Robots, Lausanne, France, 91-100, 1988.

Dai, J. S., Huang, Z., and Lipkin, H.: Mobility of overconstrained parallel mechanisms, ASME J. Mech. Des., 128, 220-222, 2006.

Di Gregorio, R.: Kinematics of the translational 3-URC mechanism, ASME J. Mech. Des., 126, 1113-1117, 2004.

Di Gregorio, R. and Parenti-Castelli, V.: Mobility analysis of the 3 UPU parallel mechanism assembled for a pure translational motion, ASME J. Mech. Des., 124, 259-264, 2002.

Fang, Y. F. and Tsai, L. W.: Analytical identification of limb structures for translational parallel manipulators, J. Robot. Syst., 21, 209-218, 2004.

Ji, P. and Wu, H. T.: Kinematics analysis of an offset 3-UPU translational parallel robotic manipulator, Robot. Auton. Syst., 42, 117 123, 2003.

Kim, D. and Chung, W. K.: Kinematic condition analysis of threeDOF pure translational parallel manipulators, ASME J. Mech. Des., 125, 323-331, 2003.

Li, W., Gao, F., and Zhang, J.: R-CUBE, A decoupled parallel manipulator only with revolute joints, Mechan. Mach. Theory, 41, 433-455, 2006.

Li, Y. M. and Xu, Q. S.: Kinematic analysis and design of a new 3-DOF translational parallel manipulator, ASME J. Mech. Des., 128, 729-737, 2006.

Li, Y. M. and Xu, Q. S.: Design and Analysis of a New 3-DOF Compliant Parallel Positioning Platform for Nanomanipulation, in: Proceedings of 5th IEEE Conference on Nanotechnology, 126129, 2005.

Li, Y. M. and $\mathrm{Xu}$, Q. S., Kinematics and dexterity analysis for a novel 3-DOF translational parallel manipulator, in: Proceedings of IEEE International Conference on Robotics and Automation, 2955-2960, 2005.

Liu, X. J., Jeong, J., and Kim, J.: A three translational DOFs parallel cube-manipulator, Robotica, 21, 645-653, 2003.

Lou, Y. J. and Li, Z. X.: A novel 3-DOF purely translational parallel mechanism, in: Proceedings of the IEEE/RSJ, International Conference on Intelligent Robots and Systems, Beijing, China, 2144-2149, 2006.

Martins, D. and Carboni, A. P., Variety and connectivity in kinematic chains, Mech. Mach. Theory, 43, 1236-1252, 2008.

Merlet, J.-P.: Jacobian, manipulability, condition number, and accuracy of parallel robots, ASME J. Mech. Des., 128, 199-206, 2006.

Ruiz-Torres, M. F., Castillo-Castaneda, E., and Briones-Leon, J. A.: Design and analysis of CICABOT: a novel translational parallel manipulator based on two 5-bar mechanisms, Robotica, 30, 449456, 2012.

Simoni, R., Doria, C. M., and Martins, D.:, Symmetry and invariants of kinematic chains and parallel manipulators, Robotica, 31, 6170, 2013.

Tsai, L. W. and Joshi, S.: Kinematics and optimization of a spatial $3-$ UPU parallel manipulator, ASME J. Mech. Des., 122, 439-446, 2000. 
Tsai, L. W. and Joshi, S.: Kinematics analysis of 3-DOF position mechanisms for use in hybrid kinematic machines, ASME J. Mech. Des., 124, 245-253, 2002.

Wang, M. F., Ceccarelli, M., and Carbone, G.: Experimental tests on operation performance of a LARM leg mechanism with 3DOF parallel architecture, Mech. Sci., 6, 1-8, doi:10.5194/ms-61-2015, 2015.
Xiao, S. L., Li, Y. M., and Meng, Q. L.: Mobility analysis of a 3PUU flexure-based manipulator based on screw theory and compliance matrix method, Int. J. Precision Eng. Manuf., 14, 13451353, 2013.

$\mathrm{Xu}, \mathrm{Q}$. S. and Li, Y. M.: A novel design of a 3-PRC translational compliant parallel micromanipulator for nanomanipulation, Robotica, 24, 527-528, 2006. 